\title{
High STAT5A Expression is Associated with Major Molecular Response Achievement Failure of Chronic Phase Chronic Myeloid Leukemia Patients Receiving Hydroxyurea before Imatinib: A Cross-sectional Study
}

\author{
Ikhwan Rinaldi* (D), Anastasia Putri ${ }^{1}$, Melva Louisa ${ }^{2}$, Sukamto Koesnoe ${ }^{3}$ \\ ${ }^{1}$ Department of Internal Medicine, Division of Hematology and Medical Oncology, Faculty of Medicine, Universitas Indonesia, \\ Cipto Mangunkusumo National General Hospital, Jakarta, Indonesia; ${ }^{2}$ Department of Pharmacology and Therapeutics, Faculty \\ of Medicine, Universitas Indonesia, Jakarta, Indonesia; ${ }^{3}$ Department of Internal Medicine, Division of Allergy and Clinical \\ Immunology, Faculty of Medicine, Universitas Indonesia, Cipto Mangunkusumo National General Hospital, Jakarta, Indonesia
}

Abstract

Edited by: Ksenija Bogoeva-Kostovska Citation: Rinaldi I, Putri A, Louisa M, Koesnoe S. Hig STAT5A Expression is Associated with Major Molecular Response Achievement Failure of Chronic Phase Chronic Myeloid Leukemia Patients Receiving Hydroxyurea before Imatinib: A Cross-sectional Study. Open Access Maced Med Sci. 2021 Sep 29; 9(B):1160-1167. ds: Imatinib: Breakpoint cluster region-ABL; STAT5A; STAT5B; Chronic myeloid leukemia
Correspondence: Ikhwan Rinaldi, Department of Internal Medicine, Division of Hematology and Medical Oncology, Cipto Mangunkusumo National General Hospital/Faculty of Medicine, Universitas Indonesia, Jakarta, Indonesia. E-mail: Ikhwan.rinaldi@ui.ac.id Received: 15-Jul-202 Revised: 17-Sep-2021 Accepted: 19-Sep-2021 Melva Louisa, Sukamto Koesno Melva Louisa, Sukamto Koesnoe
Funding: The financial support by Hibah PITTA 2018 funded by Research and Society Services Directorate, publication expenses (grant number: 233/UN.2.R3.1/ PPM.00.2018 Competing Interests: The authors have declared that no
competing interests exist Competing Interests: The authors have declared that no
competing interests exist Open Access: This is an open-access article distribution-
under the terms of the Creative Commons Attributionunder the terms of the Creative Commons Attribution-
NonCommercial 4.0 International License (CC BY-NC 4.0)
BACKGROUND: STAT5 is a transcriptional factor which when highly expressed in chronic myeloid leukemia (CML) cells stimulate proliferation and mediate resistance from tyrosine kinase inhibitors, resulting in major molecula response (MMR) failure. STAT5 has two isoforms, STAT5A and STAT5B. However, prolonged use of imatinib appears to only upregulate STAT5A pathway. In addition, the resistance conferred by STAT5A does not extend to other drugs such as hydroxyurea. Hence, STAT5A and STAT5B might have different functions in CML cells.

AIM: The objective of the study was to determine the association of STAT5A and STAT5B expression with MMR failure in CML patients.

METHODS: This was a cross-sectional study of CML patients in chronic phase with age $\geq 18$ years old, receiving IM therapy $\geq 12$ months, and previously given hydroxyurea. MMR status was evaluated and patients were categorized as achieved or failed to achieve MMR. Expression levels of STAT5A and STAT5B were conducted using RT-PCR methods. Associations between STAT5A expression, STAT5B expression, hydroxyurea duration, and imatinib duration with MMR achievement were calculated using logistic regression.

RESULTS: A total of 118 patients were analyzed; $71.1 \%$ failed to achieve MMR. Multivariate logistic regression analysis showed statistically significant association between high STAT5A expression (odds ratio [OR]: $3.852 ; 95 \%$ confidence interval [Cl]: $1.420-10.452 ; p=0.008$ ), STAT5A/STAT5B interaction (OR: $0.150 ; 95 \% \mathrm{Cl}$ : 0.038-0.593 $p=0.007$ ), longer hydroxyurea administration (OR: $3.882 ; 95 \% \mathrm{Cl}: 1.023-14.733 ; p=0.046)$, and shorter imatinib administration (OR: $0.333 ; 95 \%$ Cl: $0.132-0.840 ; p=0.020$ ) with MMR achievement failure. After adjusting STAT5A expression with STAT5A/STAT5B interaction, high STAT5A expression independently increased the likelihood of MMR achievement failure only in high expression STAT5B patients (OR: 3.852; 95\% Cl: $1.420-10.452 ; p=0.008$ ).

CONCLUSION: High STAT5A expression which is induced by high STAT5B is associated with MMR achievement failure of chronic phase CML patients who received hydroxyurea before imatinib. Longer duration of hydroxyurea and shorter duration of IM confound of STAT5A expression to MMR achievement failure.

\section{Introduction}

Chronic myeloid leukemia (CML) is one of myeloproliferative neoplasms that usually affect older adults [1]. In CML, there is a fusion of the breakpoint cluster region (BCR) gene on chromosome 22 with Abelson murine leukemia (ABL1) gene on chromosome 9 resulting in BCR-ABL1 oncogene [2]. The BCR-ABL1 oncogene has numerous signaling pathways such as RAS, RAF, JUN kinase, MYC, JAK, and STAT which can activate tyrosine kinase continuously [3]. Tyrosine kinase activation has many purposes such as maintaining survival of the cells, increasing cells proliferation, and increasing resistance against apoptosis [4]. In 1996, imatinib mesylate (IM) was discovered as the first tyrosine kinase inhibitor (TKI) and then become the first-line therapy for CML [5]. In Indonesia, IM is used for CML patients who were previously given hydroxyurea both less and more than 6 months [6].

The molecular response of IM treatment needs to be evaluated after 12 months [7]. Poor responses of IM such as failure to achieve major molecular response (MMR) are a sign of IM resistance which led to the discovery of new generations of TKls of BCR-ABL such as dasatinib, nilotinib, radotinib, ponatinib, and bosutinib or even different class of treatment [8], [9]. Several causes of IM resistance have been known such as point mutation of T315I [9], [10].

Previously, we have conducted analysis of P-glycoprotein expressions, malondialdehyde, and 
hypoxia-inducible factor 1-alpha concentrations with MMR achievement in chronic CML patients who were previously given hydroxyurea [6]. Although the study showed the lack of association between those three markers with MMR achievement, the study revealed that long-term use of hydroxyurea before IM for at least 6 months can influence MMR achievement failure through IM resistance [6]. According to a study by Warsch et al., STAT5 activation protects leukemic cells from TKIs but does not prevent cytotoxicity effect from hydroxyurea treatment which may suggest potential different pathways of hydroxyurea-induced MMR achievement failure [11].

There are many studies showing that the consequence of longer duration of IM treatment will result in the activation of STAT5A in leukemic stem cells and progenitor cells which correlated with BCR$A B L$ point mutation and increase of reactive oxygen species production [11]. In a study by Zhang et al., there are increases in STAT5A and RUNX3 expression in IM resistance [11], [12], [13]. An in vitro study by Warsch et al. showed that IM treatment for 9 days can induce increase of STAT5A expression [11]. Another in vitro study observed that STAT5B expression is also increased in IM-resistant CML [13]. Meanwhile, the effect of long-term IM use on STAT5B is still unclear. However, the study done by Zhang et al. showed STAT5A but not STAT5B as a predictor of secondary IM resistance [14]. Nevertheless, STAT5 is a potential therapeutic target as shown by higher MMR achievement from downregulation of STAT5 using STAT5 inhibitors [15], [16], [17].

To the best of our current knowledge, there are no literatures that describe effect of long-term hydroxyurea use with STAT5 activation. Hence, the role of hydroxyurea on STAT5 expression is still unknown. Therefore, this study aimed to identify the relationship between STAT5A expression and STAT5B with MMR in chronic phase CML patient on IM treatment and to analyze the effect of hydroxyurea and IM with STAT5 expression and MMR achievement failure.

\section{Materials and Methods}

\section{Study site and population}

This study is a cross-sectional study that analyzed whole blood samples obtained from previous study [6]. Patients were categorized as either achieved the MMR and did not achieve the MMR. MMR achievement is defined as the ratio of break cluster region-Abelson murine leukemia/Abelson murine leukemia (BCR-ABL/ $A B L)<0.1 \%$ which is detected by using a quantitative RT-PCR method. The expressions of STAT5A and STAT5B were examined using quantitative RT-PCR LightCycler 480 Roche $^{\mathrm{TM}}$ using Thunderbird SYBR qPCR Mix (Toyobo, Japan) kit. Quantification cycle
(Cq) was calculated automatically and the level of expression was processed with Livak's method [18]. The target gene and housekeeping gene are shown in Table 1.

Table 1: Primer sequences used in this study

\begin{tabular}{lll}
\hline Gene & Primer & Sequence \\
\hline$\beta$-actin (housekeeping gene) & Forward & 5'-GCTGGAAGGTGGACAGCGA-3' \\
& Reverse & 5'-GGCATCGTGATGGACTCCG-3' \\
STAT5A (target gene) & Forward & 5'-CATCGGTCCCTGGCTGAC-3' \\
& Reverse & 5'-AGAGGTGAAAGACCGGCAG-3' \\
STAT5B (target gene) & Forward & 5'-TCCCTGCGAGTCTGCTACT-3' \\
& Reverse & 5'-CCATTGTGTCCTCCAGATCGAA-3' \\
\hline
\end{tabular}

\section{Sample size calculation}

Sample size calculation was performed using formula for difference of proportion test. This research was never conducted in Indonesia before, hence, it is estimated that the difference in the proportion of high expression of STAT5 genes in the group that did not reach MMR and those who reached MMR was $60 \%$ and $40 \%$, respectively. As a result, the total sample size calculated was 95 patients.

\section{Inclusion and exclusion criteria}

The inclusion criteria are CML patients in chronic phase with a range of age $\geq 18$ years old, on the treatment of IM for more than 12 months, previously given hydroxyurea and MMR status available. However, patients in an acceleration or blast crisis or having IM more than $400 \mathrm{mg} /$ day and the blood sample is not appropriate for the test were excluded from this study.

\section{Statistical analysis}

Statistical analysis was conducted by using SPSS version 20.0. Continuous data were presented as mean with standard deviation (for normally distributed data) or median with minimum to maximum range (for non-normally distributed data). Categorical data were presented as numbers and percentages. Chi-square test or Fisher's exact test were used to compare categorical data and $p<0.05$ is considered to be statistically significant. Regression logistic was used for multivariate analysis. Variables chosen for regression logistic are variables which have $p<0.25$.

\section{Results}

Total 118 subjects met our inclusion and exclusion criteria, then were analyzed. There were $84(71.1 \%)$ subjects that failed to achieve the MMR and only $34(28.9 \%)$ subjects that achieved MMR. The male-to-female ratio was $1.2: 1$, with mean age $42.7 \pm 13.2$ years. Characteristics of all patients and subgroup analysis of patients who achieved the MMR 
Table 2: Baseline characteristics

\begin{tabular}{|c|c|c|c|}
\hline \multirow[t]{2}{*}{ Variables } & \multirow{2}{*}{$\begin{array}{l}\text { Total } \\
\mathrm{n}: 118\end{array}$} & \multicolumn{2}{|l|}{ MMR } \\
\hline & & Failed to achieve $n: 84$, median (min-max) & Achieved n: 34, median (min-max \\
\hline Male Sex (\%) & $53(44.9 \%)$ & $39(46.4 \%)$ & $14(41.1 \%)$ \\
\hline Age (years) (SD) & $42.77 \pm 13.207^{*}$ & $41.89 \pm 13.146$ & $44.94 \pm 13.301$ \\
\hline Duration of Imatinib Administration (months) (Median) & $18.5(12-72)$ & $17(12-72)$ & $26(12-72)$ \\
\hline Duration of Hydroxyurea Administration (months) (Median) & $2(0-168)$ & $2(0-168)$ & $1(0-24)$ \\
\hline Imatinib Dose (mg) (Median) & $400(200-400)$ & $400(200-400)$ & $400(300-400)$ \\
\hline Hemoglobin $(\mathrm{g} / \mathrm{dl})(\mathrm{SD})$ & $12.311 \pm 2.41^{\star}$ & $12.34 \pm 2.63$ & $12.24 \pm 1.82$ \\
\hline Platelet, $\left(10^{3} / \mu \mathrm{l}\right)$ (Median) & $234.5(14.4-1,232.0)$ & $240.0(14.4-1.232 .0)$ & $217.5(143-543)$ \\
\hline WBC $\left(10^{3} / \mu \mathrm{l}\right)$ (Median) & $6700(2.3-103.7)$ & $7.4(2.3-103.7)$ & $6.1(3.4-28.2)$ \\
\hline Basophil, (\%) (Median) & $0.00(0.00-47)$ & $0.00(0-47)$ & $0.00(0-5)$ \\
\hline Bands neutrophil, (\%) (Median) & $0.00(0-3)$ & $0.00(0-3)$ & $0.00(0-0)$ \\
\hline Segmented neutrophil, (\%) (Median) & $65.25 \pm 14.972^{*}$ & $63.85 \pm 15.446$ & $68.71 \pm 13.320$ \\
\hline Lymphocyte, (\%) (Median) & $25.75 \pm 12.99^{*}$ & $25.63 \pm 13.495$ & $26.06 \pm 11.835$ \\
\hline Blast, (\%) (Median) & $0(0-9)$ & $0(0-9)$ & $0(0-0)$ \\
\hline STAT5A expression (Fold over control) (Median) & $0.7374(0-867.0672)$ & $0.8873(0-867.0672)$ & $0.5284(0-59.5075)$ \\
\hline STAT5B Expression (Fold over control) (Median) & $0.3511(0-26.3549)$ & $0.2052(0-19.1597)$ & $0.4429(0-26.3549)$ \\
\hline
\end{tabular}

and did not achieved the MMR were shown in Table 2.

The overall median expression of STAT5A was 0.7374 (0.000-867.0672). The median then becomes the cutoff point to determine the subject into two groups, high expression STAT5A for subjects who have expression level more than 0.7374 and low expression in subjects who have expression level equal to less than 0.7374 .

The overall median expression of STAT5B was 0.3511 (0.000-26.3549). Similar with STAT5A, the median of STAT5B then becomes the cutoff point to determine the subject into two groups, high expression STAT5B for subjects who have expression level more than 0.3511 and low expression in subjects who have expression level equal to less than 0.3511 .

\section{Univariate analysis of association between STAT5A and STAT5B expression with MMR achievement}

Based on univariate analysis which was conducted to analyze the association between STAT5A expression with MMR achievement, no statistically significant association was observed between STAT5A expression and MMR achievement (prevalence ratio: 1.265 ; 95\% confidence interval [Cl]: 0.995-1.608; $p=0.076)$, as shown in Table 3. Meanwhile, there was a statistically significant association between STAT5B expression and MMR achievement. Patients with high expression of STAT5B have lower prevalence ratio of failed to achieve MMR than patients with low expression of STAT5B (prevalence ratio: $0.728 ; 95 \% \mathrm{Cl}: 0.570$ $0.929 ; p=0.013)$, as shown in Table 3.

Table 3: Univariate analysis of STAT5A, STAT5B, and confounding factors

\begin{tabular}{llllll}
\hline Variable & Category & \multicolumn{2}{l}{ MMR status (\%) } & $\begin{array}{l}\text { p-value } \\
\text { (continuity } \\
\text { correction) }\end{array}$ & $\begin{array}{l}\text { Prevalence } \\
\text { ratio } \\
(95 \% \mathrm{Cl})\end{array}$ \\
\cline { 3 - 4 } & & $\begin{array}{l}\text { Failed to } \\
\text { achieve }\end{array}$ & Achieved & & \\
\hline STAT5A & High & $49(79)$ & $13(21)$ & 0.076 & 1.265 \\
Expression & Low & $35(62.5)$ & $21(37.5)$ & & $(0.995-1.608)$ \\
STAT5B & High & $34(59.6)$ & $23(40.4)$ & 0.013 & 0.728 \\
Expression & Low & $50(82.0)$ & $11(18)$ & & $(0.570-0.929)$ \\
Duration of & $>6$ months & $28(90.3)$ & $3(9.7)$ & 0.012 & 1.403 \\
Hydroxyurea & $\leq 6$ months & $56(64.4)$ & $31(35.6)$ & & $(1.156-1.704)$ \\
Administration & & & & & \\
Duration of IM & $>24$ months & $24(57.1)$ & $18(42.9)$ & 0.022 & 0.724 \\
Administration & $\leq 24$ months & $60(78.9)$ & $16(21.1)$ & & $(0.544-0.964)$ \\
\hline
\end{tabular}

We also included duration of hydroxyurea and
IM administrations as variables due to being confounding factors in this study. Both of them are significant factors associated with MMR achievement based on univariate analysis (Table 3). The group that administered hydroxyurea more than 6 months has higher prevalence ratio of failed to achieve of MMR (prevalence ratio: 1.403 ; 95\% Cl: 1.156-1.704; $p=0.012$ ) (Table 3). In contrast, patients that administered IM more than 24 months have lower prevalence ratio of failed to achieve MMR when compared with IM duration equal to or less than 24 months (prevalence ratio: $0.724 ; 95 \%$ Cl: 0.544-0.964; $p=0.022$ ) (Table 3).

Univariate analysis between STAT5A expression, STAT5B expression, and confounding factors (duration of hydroxyurea and IM administrations) with MMR achievement revealed significance for all variables $(p<0.25)$ (Table 3$)$. All variables with $p<0.25$ were then subjected to multivariate analysis.

STAT5A expression, STA5B expression, STAT5A/STAT5B interaction, STAT5A/hydroxyurea interaction, STAT5A/imatinib interaction, duration of hydroxyurea administration, and duration of imatinib administration were included for multivariate analysis. Result of multivariate analysis showed significant association between STAT5A expression (odds ratio [OR]: 3.852; 95\% Cl: 1.420-10.452; $p=0.008)$, STAT5A STAT5B interaction (OR: 0.150; 95\% Cl: 0.038-0.593; $\mathrm{p}=0.007)$, duration of hydroxyurea administration more (OR: 3.882; 95\% Cl: 1.023-14.733; $p=0.046$ ), and duration of imatinib administration (OR: 0.333; 95\% Cl: 0.132-0.840; $p=0.020)$ with MMR achievement (Table 4). STAT5B, STAT5A/hydroxyurea interaction, and STAT5A/imatinib interaction failed to gain statistical significance in the multivariate analysis.

Table 4: Model 1 of association between variables with MMR achievement failure

\begin{tabular}{lllll}
\hline Independent variable & Category & OR & $95 \% \mathrm{Cl}$ & $\mathrm{p}$-value \\
\hline STAT5A expression & High & 3.852 & $1.420-10.452$ & 0.008 \\
& Low & Reference & & \\
Duration of hydroxyurea administration & $>6$ months & 3.882 & $1.023-14.733$ & 0.046 \\
& $\leq 6$ months & Reference & & \\
Duration of IM administration & $>24$ months & 0.333 & $0.132-0.840$ & 0.020 \\
& $\leq 24$ months & Reference & & \\
STAT5A/STAT5B interaction & - & 0.150 & $0.038-0.593$ & 0.007 \\
Constant & - & 0.191 & & 0.020 \\
\hline OR: Odds ratio, Cl: Confidence interval. & & & &
\end{tabular}

Due to the significant presence of STAT5A and STAT5B interaction (OR: 0.150; 95\% Cl: 0.038-0.593; 
$\mathrm{p}=0.007$ ) (Table 5), we conducted adjustment of STAT5A expression with STAT5B expression. The result is high STAT5A expression independently increased the likelihood of MMR achievement failure in high expression of STAT5B (OR: 3.852; 95\% Cl: 1.420- 10.452; $p=0.008)$. However, high STAT5A expression in low STAT5B expression had no association with MMR achievement failure (OR: 0.578; 95\% Cl: 0.054-6.196; $p=0.008)$, as shown in Table 5 .

Table 5: Adjusted STAT5A odds ratio based on STAT5B expression level

\begin{tabular}{lll}
\hline Variable & Adjusted OR & $95 \% \mathrm{Cl}$ \\
\hline High STAT5A expression in high STAT5B & 3.852 & $1.420-10.452$ \\
High STAT5A expression in low STAT5B & 0.578 & $0.054-6.196$ \\
\hline OR: Odds ratio, Cl: Confidence interval. & &
\end{tabular}

As duration of hydroxyurea and IM administrations in the first model have potential to be confounder, we analyzed several variations of multivariate analysis models to determine the best fit model. Four models were analyzed and compared based on OR differences to model 1 and level of precision to obtain the best fit regression logistic model.

The first model contains STAT5A expression, duration of hydroxyurea administration, duration of IM administration, and STAT5A/STAT5B interaction variables (Table 4). The second model contains STAT5A expression, duration of hydroxyurea administration, and STAT5A/STAT5B interaction variables (Table 6).

Table 6: Model 2 of multivariate analysis without duration of imatinib administration variable

\begin{tabular}{lllll}
\hline Independent variable & Category & OR & $95 \% \mathrm{Cl}$ & $\mathrm{p}$-value \\
\hline STAT5A expression & High & 4.222 & $1.604-11.108$ & 0.004 \\
& Low & Reference & & \\
Duration of hydroxyurea administration & $>24$ months & 3.842 & $1.036-14.243$ & 0.044 \\
& $\leq 24$ months & Reference & & \\
STAT5A by STAT5B interaction & - & 0.178 & $0.048-0.665$ & 0.010 \\
Constant & - & 0.092 & & $\leq 0.001$ \\
\hline OR: Odds ratio, Cl: Confidence interval. & & & &
\end{tabular}

The third model contains STAT5A expression, duration of IM administration, and STAT5A/STAT5B interaction variables (Table 7). The fourth model contains STAT5A expression and STAT5A/STAT5B interaction variables (Table 8). The fifth model only contains STAT5A expression variable (Table 9).

Table 7: Model 3 of multivariate analysis without the duration of hydroxyurea administration variable

\begin{tabular}{lllll}
\hline Independent variable & Category & OR & $95 \% \mathrm{Cl}$ & $\mathrm{p}$-value \\
\hline STAT5A expression & High & 4.538 & $1.712-12.033$ & 0.002 \\
& Low & Reference & & \\
Duration of IM administration & $>24$ months & 0.337 & $0.137-0.831$ & 0.018 \\
& $\leq 24$ months & Reference & & \\
STAT5A by STAT5B interaction & - & 0.119 & $0.031-0.454$ & 0.002 \\
Constant & - & 0.546 & 0.153 & \\
\hline OR: Odds ratio, Cl: Confidence interval. & & & &
\end{tabular}

Table 8: Model 4 of multivariate analysis without the duration of imatinib and hydroxyurea administration variables

\begin{tabular}{lllll}
\hline Independent variable & Category & OR & $95 \% \mathrm{Cl}$ & $\mathrm{p}$-value \\
\hline STAT5A expression & High & 4.929 & $1.913-12.697$ & 0.001 \\
STAT5A by STAT5B interaction & Low & Reference & & \\
Constant & - & 0.139 & $0.038-0.504$ & 0.003 \\
OD: Odds ratio, Cl: Confidence interval. & & 0.265 & & $\leq 0.001$ \\
\hline
\end{tabular}

The OR differences when compared with model 1 (original model) from model 2, model 3, model 4 , and model 5 are $9.6 \%, 17.8 \%, 27.95 \%$, and $41.27 \%$, respectively (Table 10). Differences of $17.8 \%, 27.95 \%$, and $41.27 \%$ are considered significant, hence model 3, model 4, and model 5 are rejected. Model 2 provides OR value which is relatively close with the OR value of model 1 (original model). Hence, both model 1 and model 2 are valid models. To determine the best fit model between model 1 and model 2, the precision of model 1 and model 2 was compared and the model with the highest precision is chosen as the final model. Based from Table 10, since model 2 has lower precision than the model 1 (9.505 vs. 9.033), model 1 is chosen as the final model.

Table 9: Model 5 of multivariate analysis without the duration of imatinib duration administration, duration of hydroxyurea administration, and STAT5A/STAT5B interaction variables

\begin{tabular}{lllll}
\hline Independent variable & Category & OR & $95 \% \mathrm{Cl}$ & p-value \\
\hline STAT5A Expression & High & 2.262 & $1.000-5.117$ & 0.050 \\
& Low & Reference & & \\
Constant & & 0.265 & - & $\leq 0.001$ \\
\hline OD: Odds ratio
\end{tabular}

OD: Odds ratio, Cl: Confidence interval.

\section{Hosmer and Lemeshow test}

Hosmer and Lemeshow test was conducted for the best fit model (Table 4) which provided $p=0.369$ (Table 11). Therefore, there is no statistically significant difference between observed MMR achievement status and expected MMR achievement status from model 1. Contingency table for Hosmer and Lemeshow test was then calculated (Table 12).

\section{Receiver operating characteristic (ROC)}

\section{curve}

After obtaining the best model from multivariate analysis, ROC curve was generated (Figure 1). The ROC analysis of the curve showed that the area under the curve was $79 \%(95 \% \mathrm{Cl}: 69.8-88.2)$ with $\mathrm{p} \leq 0.001$ (Table 13).

\section{Discussion}

The characteristics of our subject were different in the matter of age when compared with patients from western countries [19]. For example, the median age in US, France, and Germany is 66, 56, and 57 years, respectively [19]. Hence, our subjects were younger compared to the patients from western countries, as shown in Table 2. Of the total 118 subjects, the mean age was $42,77 \pm 13.207$ with the youngest age were 19 years old and the oldest were 79 years old. The previous study in Indonesia done by Reksodiputro 
Table 10: Summary of different models

\begin{tabular}{llllll}
\hline Model & Variable (s) & OR & Min & Max & Changes of OR from Model 1 (\%) \\
\hline 1 & STAT5A+Hydroxyurea+Imatinib+STAT5A*STAT5B & 3.852 & 1.420 & 10.453 & - \\
2 & STAT5A+Hydroxyurea+STAT5A*STAT5B interaction & 4.222 & 1.604 & 11.108 & $0.370(9.6 \%)$ \\
3 & STAT5A+Imatinib+STAT5A*STAT5B interaction & 4.538 & 1.721 & 12.033 & $0.686(17.8 \%)$ \\
4 & STAT5A+STAT5A*STAT5B interaction & 4.929 & 1.913 & 12.697 & $1.077(27.95 \%)$ \\
5 & STAT5A & 2.262 & 1 & 5.117 & $-1.590(41.27 \%)$ \\
\hline
\end{tabular}

Table 11: Hosmer and Lemeshow test

\begin{tabular}{llll}
\hline Step & Chi-square & df & p-value \\
\hline 1 & 7.602 & 7 & 0.369
\end{tabular}

Table 12: Contingency table for Hosmer and Lemeshow test

\begin{tabular}{lllllll}
\hline \multirow{2}{*}{ Step 1 } & \multicolumn{2}{l}{ MMR not achieved } & & \multicolumn{2}{l}{ MMR achieved } & Total \\
\cline { 2 - 3 } & Observed & Expected & & Observed & Expected & \\
\hline 1 & 5 & 5.788 & & 1 & 0.212 & 6 \\
2 & 12 & 12.225 & & 1 & 0.775 & 13 \\
3 & 12 & 10.607 & & 1.393 & 12 \\
4 & 7 & 5.807 & & 1.193 & 7 \\
5 & 26 & 25.678 & 6 & 6.322 & 32 \\
6 & 5 & 6.182 & & 4 & 2.818 & 9 \\
7 & 6 & 6.898 & 6 & 5.102 & 12 \\
8 & 7 & 7.698 & 8 & 7.302 & 15 \\
9 & 4 & 3.117 & 8 & 8.883 & 12 \\
\hline
\end{tabular}

Table 13: ROC analysis of logistic regression model and MMR achievement failure

\begin{tabular}{llll}
\hline Area & Standard error & p-value & $95 \% \mathrm{Cl}$ \\
\hline 0.790 & 0.047 & $\leq 0.001$ & $0.698-0.882$ \\
\hline
\end{tabular}

$\mathrm{Cl}$ : Confidence interval, ROC: Receiver operating characteristic.

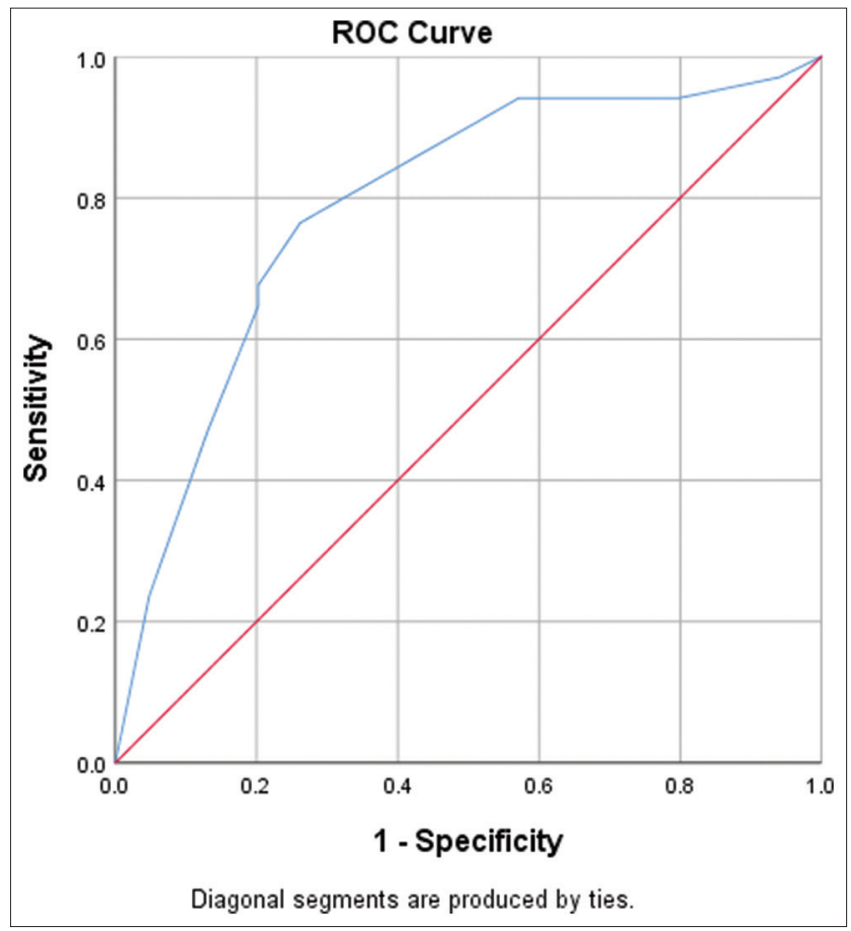

Figure 1: Receiver operating characteristic analysis of logistic regression model and major molecular response achievement failure

et al., which was conducted in 2003-2008 showed younger median of age $37(16-67)$ years [20]. The ratio of female to male in our study was 1:1.2, which are similar with other literatures [19], [20].

The level of hemoglobin and platelet in our study was in the normal range, and it was $12.31 \pm 2.41 \mathrm{mg} / \mathrm{dl}$ and $234.5(14.4-1.232 .0) 10^{3} / \mathrm{ml}$, respectively. This result was same with previous study done by Kantarjian etal. and other study by Reksodiputro, it was around $10.0-12.5 \mathrm{mg} / \mathrm{dl}$ and 303.0-424.0 $\times 10^{3} / \mathrm{ml}$, respectively [21], [22]. However, the median of leukocyte in this study was higher than normal value, it was $11,845(2.300-103.700) / \mathrm{ml}$, with $26.3 \%$ subject having leukocyte more than $10,000 / \mathrm{ml}$ after more than 12 months of IM treatment. This result was different than International Randomized Study of Interferon and STI571 (IRIS) study, which has leukocyte lower than 10,000/ml after 1 month of IM treatment [22], [23]. It means our population was in poorer condition compare to IRIS study population, possibly because of administration of hydroxyurea was more than 6 months before IM administration.

Our study used molecular response as a monitor for response after 12 months of IM therapy that has been known as (MMR; BCR-ABL1 $\leq 0.1 \%$ on the International Scale) [24]. This study reveals that after 12-month treatment of IM, $28.8 \%$ (n: 34 ) subjects achieve the MMR. The result was in the range of previous study, between $18 \%$ and $58 \%$ [25]. The highest proportion of patients who achieve the MMR was observed in IRIS study, after 8 years, $86 \%$ patients achieve the MMR [22], [23]. The difference between the proportion of our study and IRIS study can be possibly explained from the use of hydroxyurea administration before IM administration more than 6 months in our study which translated to higher IM resistance in our population than IRIS study.

The mechanism of resistance to IM based on molecular basis was differentiated into three major mechanisms. Two of the mechanisms affect the BCR$A B L$ gene such as mutation in tyrosine kinase domain and overexpression of $B C R-A B L$ protein while the third mechanism is independent to BCR-ABL gene, which also includes overexpression of STAT5 [26], [27]. The overexpression of STAT5 shown a role of IM resistance by activation of $A B L-X L$ as antiapoptotic, gain of uncontrolled proliferation, and angiogenesis ability [26], [28].

The aim of our study is to identify the association between expression levels of STAT5A and STAT5B with MMR achievement in chronic phase CML patients on IM treatment. In the univariate analysis, we observe that no statistically significant association was between STAT5A expression and MMR achievement failure (prevalence ratio: $1.265 ; 95 \% \mathrm{Cl}$ : 0.995-1.608; $p=0.076$ ). Meanwhile, high expression of STAT5B is as a protective factor to MMR achievement failure (prevalence ratio: $0.728 ; 95 \% \mathrm{Cl}$ : $0.570-0.929$; $p=0.013)$.

This study also identifies the role of hydroxyurea 
and IM administration in MMR achievement failure. In the univariate analysis, longer hydroxyurea ( $>6$ months) administration was associated with MMR achievement failure (prevalence ratio: $1.403 ; 95 \% \mathrm{Cl}: 1.156-1.704$; $\mathrm{p}=0.012)$ and longer IM (>24 months) administration was associated with successful MMR achievement (prevalence ratio: $0.724 ; 95 \% \mathrm{Cl}$ : $0.544-0.964$; $p=0.022$ ), as shown in Table 3 . This result is inherent with previous study that showed hydroxyurea more than 6 months which was associated with MMR achievement failure as well [6].

In the final model of multivariate
analysis, associated with MMR achievement failure (OR: 3.852; 95\% Cl: 1.420- 10.452; $p=0.008$ ) and STAT5B has no contribution to MMR achievement failure (Table 4). We also measured the interaction between STAT5A and STAT5B. The result of the interaction showed that STAT5A is only associated with MMR achievement failure when in high STAT5B expression patients (OR: $3.852 ; 95 \% \mathrm{Cl}: 1.420-10.452$ ). If the patients have low STAT5B expression, then STAT5A is not associated with MMR achievement (OR: 0.578; 95\% Cl: 0.054-6.196). Variations of the models were analyzed to obtain the best multivariate analysis model (Table 10). The result showed that the original model is the best model for MMR achievement failure.

The result of multivariate analysis is also supported by Hosmer and Lemeshow test which showed no significant difference between the model prediction and observed events (Table 11). Finally, ROC analysis has AUC value of $79 \%$ (95\% Cl: $69.8-88.2)$ which is moderately accurate in differentiating between patients that achieve MMR and those that fail to achieve MMR.

This finding also supports the hypothesis that high expression STAT5A has a role in CML IM resistance and is in concordance with other studies such as the study done by Warsch et al., which found higher mean of STAT5A expression in the IM resistance subjects than in IM sensitive subjects [11], [14]. However, expression in STAT5B shows opposite result with STAT5A. Again, this is similar with other studies [14], [29].

There are several differences of functions between STAT5A and STAT5B such as differences in responses to stress and additionally, in $v-A B L$ expressed cells STAT5B can induce STAT5A expression but STAT5A cannot induce STAT5B expression [30], [31], [32]. Study by Schönitz et al. showed that in the presence of BCR-ABL, cytoplasmic retention of STAT5A but not STAT5B is observed [29]. The same study also showed that the interaction of STAT5B-BCR-ABL was required for human cells proliferation [29].

The hypothesis for the cause of lower STAT5B expression in CML patients who failed to achieve MMR compared to STAT5A is due to the major role of STAT5B in primitive hematopoietic cells while STA5A has more role in TKI resistance CML [14]. The other hypothesis is the presence of STAT5B mutation which makes it difficult to detect the gene expression of STAT5B.

In the final model, hydroxyurea use of more than 6 months is associated with failure of MMR achievement, but IM use of more than 24 months associated with successful MMR achievement. Therefore, in our study, failure of MMR achievement was caused by administration of hydroxyurea, not by IM.

The exact mechanism of how longer duration of hydroxyurea administration can cause MMR achievement failure might be caused by reactive oxygen species production during hydroxyurea metabolism in the body, however, our previous study showed no differences in P-glycoprotein expressions, malondialdehyde, and hypoxia-inducible factor 1-alpha concentrations after hydroxyurea use [6]. Elimination of IM duration variables from final models causes an increase of STAT5A OR of more than $10 \%$. It means that longer duration of hydroxyurea administration can increase STAT5A and affects MMR achievement failure. Elimination of hydroxyurea duration variables from final models also causes an increase of STAT5A OR of more than $10 \%$, similar with hydroxyurea. This also means that longer duration of IM administration can increase STAT5A and affects MMR achievement failure. It can be inferred that both hydroxyurea and IM duration are confounding factors.

Finally, the duration of hydroxyurea and IM administration were included in the final model of MMR achievement failure. In clinical practice, hydroxyurea administration is almost always given before IM. Administration of hydroxyurea was causing an increase of STAT5A, then effect to MMR achievement failure. After IM administration, the effect of STAT5A to MMR achievement failure was decreased ( $O R=4222$ vs. 3852). This final model showed that the duration of hydroxyurea can cause MMR achievement failure through STAT5A expression.

\section{Study limitation}

There were several limitations in this study. The first limitation was lack of IM duration and hydroxyurea duration restriction in our subjects. Second, there were differences in IM and hydroxyurea treatment duration. Hence, it is possible that there may be a confounding in the analysis. In addition, the time of MMR measurement conducted was different between each patient, which may cause heterogeneity in outcome measurement. Finally, this study has the usual limitations of a cross-sectional study. 


\section{Conclusion}

In conclusion, the higher expression of STAT5A may be used as a potential marker of MMR achievement failure in chronic phase CML patient who received hydroxyurea before IM administration more than 12 months. High expression of STAT5B is shown to induce high STAT5A expression. An inhibitor of STAT5B might be beneficial as it may indirectly inhibit STAT5A to overcome of IM resistance in our study. Longer duration of hydroxyurea and IM confound STAT5A expression to MMR achievement failure. Research with cohort study design is needed to confirm our findings of the role of STAT5A and STAT5B in CML.

\section{Acknowledgment}

The authors would like to express their gratitude to Kevin Winston MD MRes for his help in data analysis and manuscript revision.

\section{Ethical Statements}

This study protocol as well as the written informed consent form has been reviewed and approved by the Ethical Committee of the Faculty of Medicine, Universitas Indonesia (Certificate of ethical approval 1053/UN2.F1/ETIK/2018). All patients were given explanation about the study and provided written informed consent before the interview.

\section{Availability of Data and Material}

The datasets used and/or analyzed during the current study are available from the corresponding author on reasonable request.

\section{Author Contributions}

Anastasia Putri, Ikhwan Rinaldi, Melva Lousia, and Sukamto Koesno conceived of the presented idea, developed the theory, performed the computations, and verified the analytical methods. Anastasia Putri and
Ikhwan Rinaldi wrote the manuscript with support and collaboration from Melva Lousia and Sukamto Koesno. All authors discussed the results and contributed to the final manuscript. Ikhwan Rinaldi is the corresponding author.

\section{References}

1. Granatowicz A, Piatek Cl, Moschiano E, El-Hemaidi I, Armitage JD, Akhtari M. An overview and update of chronic myeloid leukemia for primary care physicians. Korean $\mathrm{J}$ Fam Med. 2015;36(5):197-202. http://doi.org/10.4082/ kjfm.2015.36.5.197

PMid:26435808

2. Apperley JF. Chronic myeloid leukaemia. Lancet Lond Engl. 2015;385(9976):1447-59. http://doi.org/10.1016/ S0140-6736(13)62120-0

PMid:25484026

3. Jabbour E, Kantarjian H. Chronic myeloid leukemia: 2018 update on diagnosis, therapy and monitoring. Am J Hematol. 2018;93(3):442-59. http://doi.org/10.1002/ajh.25011 PMid:29411417

4. Deininger MW, Vieira S, Mendiola R, Schultheis B, Goldman JM, Melo JV. BCR-ABL tyrosine kinase activity regulates the expression of multiple genes implicated in the pathogenesis of chronic myeloid leukemia. Cancer Res. 2000;60(7):2049-55. PMid:10766197

5. Casetti L. New Roles of STAT5 Factors in Chronic Myeloid Leukemia Cell Maintenance, No. 166; 2014.

6. Rinaldi I, Reksodiputro AH, Jusman SW, Harahap A Setiabudy R, Wanandi SI, et al. Longer hydroxyurea administration prior to imatinib mesylate is risk factor for unsuccessful major molecular response in chronic-phase chronic myeloid leukemia: Possibility of p-glycoprotein role. Asian Pac J Cancer Prev. 2019;20(12):3689-95. https://doi. org/10.31557/APJCP.2019.20.12.3689

PMid:31870110

7. Rizzieri D, Moore JO. Implementation of management guidelines for chronic myeloid leukemia. Pharm Ther. 2012;37(11):640-8.

PMid:23204819

8. Wei G, Rafiyath S, Liu D. First-line treatment for chronic myeloid leukemia: Dasatinib, nilotinib, or imatinib. J Hematol Oncol. 2010;3:47. https://doi.org/10.1186/1756-8722-3-47 PMid:21108851

9. Valent $\mathrm{P}$. Imatinib-resistant chronic myeloid leukemia $(\mathrm{CML})$ Current concepts on pathogenesis and new emerging pharmacologic approaches. Biol Targets Ther. 2007;1(4):433-48. PMid:19707313

10. Legros L, Hayette S, Nicolini FE, Raynaud S, Chabane K, Magaud JP, et al. BCR-ABL T315I transcript disappearance in an imatinib-resistant $\mathrm{CML}$ patient treated with homoharringtonine: A new therapeutic challenge? Leukemia. 2007;21(10):2204-6. https://doi.org/10.1038/sj.leu.2404772 PMid:17541396

11. Warsch W, Kollmann K, Eckelhart E, Fajmann S, Cerny- Reiterer S, Hölbl A, et al. High STAT5 levels mediate imatinib resistance and indicate disease progression in chronic myeloid leukemia. Blood. 2011;117(12):3409-20. https://doi. org/10.1182/blood-2009-10-248211

PMid:21220747 
12. Dorritie KA, McCubrey JA, Johnson DE. STAT transcription factors in hematopoiesis and leukemogenesis: Opportunities for therapeutic intervention. Leukemia. 2014;28(2):248-57. https:// doi.org/10.1038/leu.2013.192

PMid:23797472

13. Cheng Y, Hao Y, Zhang A, Hu C, Jiang X, Wu Q, et al. Persistent STAT5-mediated ROS production and involvement of aberrant p53 apoptotic signaling in the resistance of chronic myeloid leukemia to imatinib. Int J Mol Med. 2018;41(1):455-63. https:// doi.org/10.3892/ijmm.2017.3205

PMid:29115375

14. Zhang WW, Cortes JE, Yao H, Zhang L, Reddy NG, Jabbour E, et al. Predictors of primary imatinib resistance in chronic myelogenous leukemia are distinct from those in secondary imatinib resistance. J Clin Oncol. 2009;27(22):3642-49. https:// doi.org/10.1200/JCO.2008.19.4076

PMid:19506164

15. Rousselot P, Roy L, Etienne G, Legros L, Charbonnier A, Coituex V, et al. Targeting STAT5 expression resulted in molecular response improvement in patients with chronic phase CML treated with imatinib. Blood. 2012;120(21):696-6. https:// doi.org/10.1182/blood.V120.21.696.696

16. Tolomeo M, Meli M, Grimaudo S. STAT5 and STAT5 inhibitors in hematological malignancies. Anticancer Agents Med Chem. 2019;19(17):2036-46. http://doi.org/10.2174/187152061966619 0906160848

PMid:31490767

17. Gleixner KV, Schneeweiss M, Eisenwort G, Berger D, Herrmann $\mathrm{H}$, Blatt $\mathrm{K}$, et al. Combined targeting of STAT3 and STAT5: A novel approach to overcome drug resistance in chronic myeloid leukemia. Haematologica. 2017;102(9):1519- 29. http:// doi.org/10.3324/haematol.2016.163436 PMid:28596283

18. Livak KJ, Schmittgen TD. Analysis of relative gene expression data using real-time quantitative PCR and the 2(-Delta Delta C(T)) Method. Methods San Diego Calif. 2001;25(4):402-8. http://doi.org/10.1006/meth.2001.1262

PMid:11846609

19. Höglund $M$, Sandin $F$, Simonsson B. Epidemiology of chronic myeloid leukaemia: An update. Ann Hematol. 2015;94(Suppl 2):S241-7. http://doi.org/10.1007/ s00277-015-2314-2

PMid:25814090

20. Reksodiputro AH, Syafei S, Prayogo N, Karsono B, Rinaldi I, Rajabto W, et al. Clinical characteristics and hematologic responses to Imatinib in patients with chronic phase myeloid leukemia (CML) at Cipto Mangunkusumo Hospital. Acta Med Indones. 2010;42(1):2-5.

PMid:20305324

21. Kantarjian H, Sawyers C, Hochhaus A, Guilhot F, Schiffer C, Gambacorti-Passerini C, et al. Hematologic and cytogenetic responses to imatinib mesylate in chronic myelogenous leukemia. N Engl J Med. 2002;346(9):645-52. http://doi. org/10.1056/NEJMoa011573

PMid:11870241

22. O'Brien SG, Guilhot F, Larson RA, et al. Imatinib compared with interferon and low-dose cytarabine for newly diagnosed chronic-phase chronic myeloid leukemia. $\mathrm{N}$ Engl J Med.
2003;348(11):994-1004. http://doi.org/10.1056/NEJMoa022457 PMid: 12637609

23. Deininger M, O'Brien SG, Guilhot F, Goldman JM, Hochhause A Hughes TP, et al. International randomized study of interferon Vs STI571 (IRIS) 8-year follow up: Sustained survival and low risk for progression or events in patients with newly diagnosed chronic myeloid leukemia in chronic phase (CML-CP) treated with imatinib. Blood. 2009;114(22):1126-6.

24. Renault IZ, Scholl V, Hassan R, Capelleti P, de Lima M, Cortes J. The significance of major and stable molecular responses in chronic myeloid leukemia in the tyrosine kinase inhibitor era. Rev Bras Hematol. 2011;33(6):455-60. http://doi. org/10.5581/1516-8484.20110122 PMid:23049363

25. Sacha T. Imatinib in chronic myeloid leukemia: An overview. Mediterr J Hematol Infect Dis. 2014;6(1):e2014007. http://doi. org/10.4084/MJHID.2014.007

PMid:24455116

26. Melo JV, Chuah C. Resistance to imatinib mesylate in chronic myeloid leukaemia. Cancer Lett. 2007;249(2):121-32. http://doi. org/10.1016/j.canlet.2006.07.010

PMid:16949736

27. Hochhaus A, Kreil S, Corbin AS, La Rosée P, Müller MC, Lahaye $\mathrm{T}$, et al. Molecular and chromosomal mechanisms of resistance to imatinib (STI571) therapy. Leukemia 2002;16(11):2190-6. http://doi.org/10.1038/sj.leu.2402741

PMid:12399961

28. Kaymaz BT, Selvi N, Gündüz $C$, Aktan $C$, Dalmızrak $A$ Saydam G, et al. Repression of STAT3, STAT5A, and STAT5B expressions in chronic myelogenous leukemia cell line K-562 with unmodified or chemically modified siRNAs and induction of apoptosis. Ann Hematol. 2013;92(2):151-62. http://doi. org/10.1007/s00277-012-1575-2

PMid:23053176

29. Schaller-Schönitz M, Barzan D, Williamson AJ, Griffiths JR, Dallmann I, Battmer K, et al. BCR-ABL Affects STAT5A and STAT5B Differentially. PLoS One. 2014;9(5):e97243. http://doi. org/10.1371/journal.pone.0097243 PMid:24836440

30. Mi T, Wang Z, Bunting KD. The cooperative relationship between STAT5 and reactive oxygen species in leukemia: Mechanism and therapeutic potential. Cancers. 2018;10(10):359. http://doi. org/10.3390/cancers 10100359

PMid:30262727

31. Casetti L, Martin-Lannerée S, Najjar I, Plo I, Augé S, Roy L, et al. Differential contributions of STAT5A and STAT5B to stress protection and tyrosine kinase inhibitor resistance of chronic myeloid leukemia stem/progenitor cells. Cancer Res. 2013;73(7):2052-8. http://doi.org/10.1158/0008-5472. CAN-12-3955 PMid:23400594

32. Kollmann S, Grundschober E, Maurer B, Warsch W, Grausenburger R, Edlinger $\mathrm{L}$, et al. Twins with different personalities: STAT5B but not STAT5A has a key role in BCR/ ABL-induced leukemia. Leukemia. 2019;33(7):1583-97. http:// doi.org/10.1038/s41375-018-0369-5

PMid:30679796 